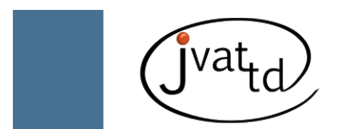

\title{
Symptom patterns in adult patients stung by scorpions with emphasis on coagulopathy and hemoglubinuria
}

Rahmani AH (1), Jalali A (2)

(1) Department of Clinical Toxicology, Razi Hospital, School of Medicine and Toxicology Research Center, Jundishapur University of Medical Sciences, Ahvaz, Iran; (2) Department of Pharmacology and Toxicology, School of Pharmacy and Toxicology Research Center, Jundishapur University of Medical Sciences, Ahvaz, Iran.

\begin{abstract}
The aim of this retrospective descriptive study was to highlight clinical manifestations $24-48$ hours following referral of adult patients stung by scorpions. This study contains clinical records of 290 patients admitted to Razi Hospital due to scorpion stings in Ahvaz, Khuzestan province from 2004 to 2005. The most prevalent patient age range was $15-20$ years (30.3\%). The most common sting location (41.3\%) was the upper extremity; nearly half (49.6\%) had been admitted within 6-24 hours following sting, while a large majority (85.5\%) were hospitalized for $24-48$ hours. A total of $116(40 \%)$ patients presented hemoglobinuria. Contrary to available prior reports, the symptoms in none of the patients were accompanied by neurological manifestations. Kidney manifestations (BUN, creatinine), coagulopathy and transfusion were observed in patients with blood cell lysis and hemoglobinuria. The kidney problems were seen more in patients who had been admitted more than 24 hours after the accident. Overall, the findings demonstrate that coagulation and hemoglobinuria signs produced by scorpion sting in Ahvaz differ significantly from those reported elsewhere.
\end{abstract}

Key words: scorpion, stings, signs and symptoms, coagulopathy, hemoglobinuria.

\section{INTRODUCTION}

Scorpion stings constitute one of the medically important problems worldwide in tropical and subtropical regions, especially in hot climate areas such as Khuzestan province in the southwest region of Iran (1). Well-known scorpion species of Iran are distributed among five genera including Mesobuthus, Compsobuthus, Hottentotta (Buthotus), Orthochirus, Androctonus and Hemiscorpius, which have been reported (as stinging scorpions) in Khuzestan province (2). Most of the deaths due to scorpion stings in Iran have been caused by Hemiscorpius lepturus ( $H$. lepturus) (3).

Because scorpions do not need to burrow into the ground for continued survival and shelter, so they can be found in the shade under rocks, homes and elsewhere. Specimens of the $H$. lepturus genus were caught in various Iranian provinces such as Khuzestan, Fars, Lorestan, Bushehr and Bandar Abbas, but they mostly have been observed in Khuzestan cities such as Ramhormoz, Baghmalek, Masjed Soleyman, Izeh and Ahvaz.

Clinical symptoms and mortality from a scorpion sting are related to various factors including season, age of patient, the location of sting, history of cardiopulmonary disease and allergic systemic reactions to drugs and toxins $(4,5)$. Pain, cellulitis, necrosis, blisters and skin irritation and itching are among the local symptoms, whereas systemic symptoms include blood cell lysis, hemoglobinuria, vascular disorders, renal failure, pulmonary edema especially in children, and some neurological symptoms like sweating, restlessness, changes in blood pressure and muscle problems $(6,7)$. The 
symptoms of stings from other scorpion species including Mesobuthus eupeus (Stinging has fairly severe pain) are not similar to H. lepturus (painless), but local types are more severe and systemic types are less so. In case of the black scorpion Androctonus crassicauda the sting is accompanied by such additional neuromuscular symptoms as seizures, shivering, clonus and tetanus, while the patient also suffers from severe pain (3).

Coagulopathy and hemoglobinuria are rare manifestations of scorpion envenoming. Therefore the aim of this study was to evaluate scorpion-sting patients who presented at hospital admission with signs and symptoms ranging from hemoglobinuria, coagulopathy, blood transfusion to life-threatening renal failure.

\section{MATERIALS AND METHODS}

This is a retrospective study of the clinical records of 290 patients who were referred to Razi hospital due to scorpion stings in Ahvaz from 2004 to 2005 . All patients were aged up to 11 years (Table 1). Data records in compliance with all ethical standards and without mentioning names and personal details of patients were retrieved and entered in the designed checklist that includes the following variables: age, sex, time and location of sting, onset of symptoms after the sting, the need for transfusion, coagulation disorder, hemoglobinuria, urinary volume changes, and changes in kidney tests such as BUN (blood, urea, and nitrogen) and creatinine, neurological symptoms (like severe pain, seizures, sweating and muscle spasms), duration of hospitalization and death.

The empirical guideline deals with investigation of blood on dipstick urine following an unknown yellow scorpion sting with macroscopic and microscopic analyses. Preliminary diagnosis of hemoglobinuria is made on red urine. Centrifugation aids in the differential diagnosis. In the present study, hemoglobinuria was confirmed when few red blood cells (RBCs) were seen microscopically (in combination with dipstick analysis). The hematological tests were carried out using an automated cell counter (Coulter T890) to quantify erythrocytes, leukocytes and platelets. Coagulopathy was defined as the presence of either a platelet count $<150,000 / \mathrm{mm}^{3}$, prothrombin time above normal, or fibrinogen level $<150 \mathrm{mg} / \mathrm{dL}$ (8). The patients received blood transfusion when their hemoglobin level was below 8 grams per deciliter (g/dL).

This study was approved by the Institutional Ethics Committee of the School of Medicine, Ahvaz Jundishapur University of Medical Sciences.

\section{Statistical analysis}

SPSS (Statistical Package for Social Sciences) version 16 with descriptive (frequencies) and analytic tests ( $t$ test) was used to analyze data and determine the significant differences. The variables were compared by the $\chi^{2}$ test. A difference of $p<0.05$ is considered statistically significant.

\section{RESULTS}

A total of 290 patients [139 (47.9\%) male and $151(52.1 \%)$ women] were studied. The largest proportion of the patients $(30.3 \%)$ was in the age range of $15-20$ years (Table 1 ). The most prevalent sting location (41.3\%) was in the upper extremities. Almost half of the patients (49.6\%) had been admitted to hospital within 6-24 hours after the sting followed by $13.1 \%$ who were referred within less than six hours. The scorpion stung most frequently at night [142 (48.9\%) cases] while the least common time frame was midday (4.8\%). In 230 patients the scorpion types were unknown, while 17 (5.8\%) cases were attributed to $H$. lepturus. The most common geographic locations of scorpion-sting cases were in Ramhormoz with 41(14.1\%) patients and in the Golestan region in Ahvaz with 45 (15.5\%) patients. A total of $116(40 \%)$ patients were suffering from hemoglobinuria. Among patients with hemoglobinuria signs, 4 (3.4\%) and 9 cases (7.75\%) were stung by $A$. crassicauda and $H$. lepturus scorpion, respectively. Eleven (9.4\%) and 92 cases $(79.3 \%)$ were stung by a yellow scorpion (unknown species) and unknown scorpion, respectively. Hemoglobinuria was found in 44\% and $52.9 \%$ of $A$. crassicauda and H. lepturus cases, respectively. The relationship between the type of scorpion and hemoglobinuria was not significant. The times at which hemoglobinuria occurred in 11 (9\%), 58 (50\%) and 47 (41\%) cases were less than 6, between 6-24 and more than 24 hours prior to admission, respectively. The relationship between the time of occurrence and hemoglobinuria was 
Table 1. Age distribution of 290 cases of adult patients stung by scorpions in Khuzestan, Iran

\begin{tabular}{c|c|c}
\hline Age (years) & Number of patients & Percentage \\
\hline $11-14$ & 22 & 7.58 \\
\hline $15-20$ & 88 & 30.3 \\
\hline $21-30$ & 76 & 26.2 \\
\hline $31-40$ & 48 & 16.55 \\
\hline $41-50$ & 23 & 7.93 \\
\hline $51-60$ & 22 & 7.58 \\
\hline $61-70$ & 6 & 2 \\
\hline $71-80$ & 5 & 1.72 \\
\hline Total & 290 & 100 \\
\hline
\end{tabular}

significant $(\mathrm{p}<0.05)$. Twenty-one subjects were also suffering from coagulopathy disorder. Of the 30 patients who required blood transfusion, 25 individuals had received blood due to hemoglobinuria while the rest needed other blood products. All three patients who died not only had been stung by the scorpion $H$. lepturus but also suffered from hemoglobinuria, coagulopathy and an insufficiency of at least one blood product. Out of 290 patients, 248 $(85.5 \%)$ were hospitalized for $24-48$ hours. The onset of symptoms in none of the patients was accompanied by neurological complications (such as seizures, sweating and muscle spasms) and no patients suffered from neurological complications during hospitalization.

In the results section only the highlights of clinical features and relevant laboratory findings in 290 patients envenomed by scorpion are indicated.

\section{DISCUSSION}

In the present study, some parameters such as age, sex, location and the symptoms at the time of hospital admission were similar to studies that were conducted in Saudi Arabia (9) and Turkey (10), but post-sting referral time differed between our country and Mexico (11). In Mexico, the majority had been admitted within one hour post-sting, while the most prevalent time frame in the present study was between 6-24 hours. This difference may be due to the painlessness and late referral of $H$. lepturus envenoming (6). The late referral may be due to few and mild early signs provoked by the H. lepturus sting. Furthermore, the sting of Mesobuthus eupeus can be serious, but does not produce severe pain or signs for an urgent referral.

But it appears there are a few very important results in this study, which in fact were considered to be its primary goals. First, in this study the symptoms in none of the patients were accompanied by neurological complications, from which no patients were suffering during the hospitalization. This is in contrast to previous studies conducted in Iran and other countries, which repeatedly reported neurological symptoms in scorpion-sting patients during hospitalization and considered such symptoms to be the major signs $(6,12-14)$. All patients in this study were past age 11, while in Pipelzadeh and colleagues study, the majority of cases were children aged less than 12 years. Resolving this controversy is somewhat difficult and requires further research in other areas. Second, it was previously reported that kidney impairment or failure due to scorpion envenoming, especially in the case of H. lepturus is one of the early symptoms and the scorpion venom is toxic $(6,15-17)$. While in the present study, kidney problems were present only in patients with blood cell lysis and hemoglobinuria. Third, hemoglobinuria, coagulopathy, transfusion and kidney problems were seen more in patients who had been admitted after 24 hours following the sting. These problems were more frequently provoked by $H$. lepturus than the other scorpions. All three patients who died also had been stung by $H$. lepturus and were delayed in referral.

In the present study coagulation disorder 
and hemoglobinuria were observed more often than in the available reports. In these conditions transfusion was essential. Also, the three patients who died had presented coagulation disorders and hemoglobinuria and received transfusion. Therefore, these signs are indicative of a poor prognosis following scorpion sting. Furthermore, early recognition of these conditions in hospitalized adult patients is necessary.

As had been predicted, the symptoms presented greater severity following $H$. lepturus envenoming while any delay in referral would put the patient at risk of death. Scorpion toxins are proteins with variable molecular weight, generally less than 7-8 $\mathrm{kDa}$. These toxins target specific receptors, mostly on cell membranes and manifest different dose-dependent signs, mainly neurological, cardiovascular and muscular ones $(18,19)$. The scorpion venom neurotoxins are mostly the presynaptic and postsynaptic curarelike neurotoxins. A newly isolated toxin within H. lepturus venom, Heminecrolysin, a $33 \mathrm{kDa}$ protein endowed with sphingomyelinase $\mathrm{D}$, hemolytic and dermonecrotic activities, exceeds the size of the scorpion venom toxins by 5 -fold (20). So it is likely that $H$. lepturus venom possesses various structures and activities compared with scorpion venom neurotoxins. Therefore, it may be concluded that at least a fraction of this venom causes damage to the vascular endothelium or induces a cytolysis such as destruction of red blood cells. Heminecrolysin has a higher molecular weight than those of scorpion toxins, from which it is distinguished by its catalytic properties. H. lepturus venom is rich in enzymes. The pharmacological effects of enzymes may depend more on the duration of the enzymatic reaction cycle (21). Clinical manifestations following $H$. lepturus envenoming such as late renal failure are therefore mainly time-dependent. The other probable mechanism that mediates blood cell damage is complement hemolysis or sphingomyelinase $(22,23)$. Furthermore, the coagulopathy described in the present study, characterized principally by low levels of plasma fibrinogen and coagulation factors, can be explained by the thrombinlike enzyme activity from $H$. lepturus venom as shown in human envenoming caused by rattlesnakes (24). However, the alterations in the levels of coagulation factors, thrombinantithrombin complex and other characteristics of physiopathology of coagulopathy are difficult to explain as a direct effect of $H$. lepturus venom.

Fourth, all the patients were immediately administered one or two polyvalent anti-venoms intramuscularly upon hospital admission; but despite this many signs and symptoms, in some cases severe, had been observed. However, the method of anti-venom administration is now questioned, which may be is due to the duration of post-sting administration, the improper dosage or other unknown causes. Currently, the scorpionsting victims in Iran, including those envenomed by $H$. lepturus, are treated in most clinical centers with intramuscular administration of available antivenom. As suggested in the available protocol, 2 vials of $5 \mathrm{~mL}$ of antivenom are recommended for an adult patient. The majority of clinical centers are concerned with the effects of serum sickness following intravenous administration and routinely use antivenom by means of intramuscular route.

Since the antivenom should be administered immediately following such a sting, these findings further confirm that the intramuscular route is a suitable means of administration against $H$. lepturus envenoming with late referral to clinical centers.

This is a retrospective and hospital information-based study and the cases that had been registered and what seen in clinical practice have been analyzed. Therefore, changes in symptom patterns differing from those previously reported and poor effectiveness of anti-venom administration in late referral of adult scorpionsting patients are described. Furthermore, more detailed research is required, especially in the field of scorpion genetics, on changes in enzymes and toxins found in scorpion venoms, and their effects on human organs as well as appropriate dose, the best duration for administration of antivenom and the treatment protocols.

\section{ACKNOWLEDGMENTS}

The authors thank the staff of Razi hospital for their help in carrying out the study. This study was supported by the of Jundishapur University.

\section{COPYRIGHT}

(C) CEVAP 2012

\section{SUBMISSION STATUS}

Received: December 20, 2012. 
Accepted: April 5, 2012.

Abstract published online: April 5, 2012.

Full paper published online: November 30, 2012.

\section{CONFLICTS OF INTEREST}

The authors declare no conflicts of interest.

\section{FINANCIAL SOURCE}

The Ahvaz Jundishapur University of Medical Sciences, Ahvaz, Iran provided the financial grants.

\section{ETHICS COMMITTEE APPROVAL}

The present study was approved by the Institutional Ethics Committee of the School of Medicine, Ahvaz Jundishapur University of Medical Sciences (Ref. n. IA/1630).

\section{CORRESPONDENCE TO}

Amir Jalali, Golestan Road, School of Pharmacy, Jundishapur University of Medical Sciences, Ahvaz, Iran. Phone: +98 611 3738380. Fax: +98 611 3738381.Email: amjalali@hotmail.com.

\section{REFERENCES}

1. ChippauxJP, Goyffon M. Epidemiology of scorpionism: a global appraisal. Acta Trop. 2008;107(2):71-9.

2. Dehghani R, Djadid ND, Shahbazzadeh D, Bigdelli S. Introducing Compsobuthus matthiesseni (Birula, 1905) scorpion as one of the major stinging scorpions in Khuzestan, Iran. Toxicon. 2009;54(3):272-5.

3. Radmanesh M. Clinical study of Hemiscorpion lepturus in Iran. J Trop Med Hyg. 1990;93(5):327-32.

4. Pucca MB, Roncolato EC, Campos LB, Fernandes FA, Mendes GR, Bertolini TB, et al. Experimental Tityus serrulatus scorpion envenomation: age-and sex related differences in symptoms and mortality in mice. J Venom Anim Toxins incl Trop Dis. 2011;17(3):325-32.

5. Bahloul M, Chaari A, Dammak H, Samet M, Chtara K, Chelly $\mathrm{H}$, et al. Pulmonary edema following scorpion envenomation: Mechanisms, clinical manifestations, diagnosis and treatment. Int J Cardiol. 2011 Nov 8. [Epub ahead of print]. Available at: http://dx.doi. org/10.1016/j.ijcard.2011.10.013.

6. Pipelzadeh MH, Jalali A, Taraz M, Pourabbas R, Zaremirakabadi A. An epidemiological and clinical study on scorpionism by the Iranian scorpion Hemiscorpius lepturus. Toxicon. 2007;50(7):984-92.

7. Radmanesh M. Cutaneous manifestations of the Hemiscorpius lepturus sting: a clinical study. Int J Dermatol. 1998;37(7):500-7.

8. Bogdan GM, Dart RC, Falbo SC, McNally J, Spaite D. Recurrent coagulopathy after antivenom treatment of crotalid snakebite. South Med J. 2000;93(6):562-6.

9. Al-Sadoon MK, Jarrar M. Epidemiological study of scorpion stings in Saudi Arabia between 1993 and 1997.
J Venom Anim Toxins incl Trop Dis. 2003;9(1):54-64.

10. Adiguzel S, Ozkan O, Inceoglu B. Epidemiological and clinical characteristics of scorpionism in children in Sanliurfa, Turkey. Toxicon. 2007;49(6):875-80.

11. Chowell G, Díaz-Dueñas P, Bustos-Saldaña R, Mireles AA, Fet V. Epidemiological and clinical characteristics of scorpionism in Colima, Mexico (2000-2001). Toxicon. 2006;47(7):753-8.

12. Nouira S, Boukef R, Nciri N, Haguiga H, Elatrous S, Besbes L, et al. A clinical score predicting the need for hospitalization in scorpion envenomation. Am J Emerg Med. 2007;25(4):414-9.

13. Bahloul M, Chabchoub I, Chaari A, Chtara K, Kallel $\mathrm{H}$, Dammak $\mathrm{H}$, et al. Scorpion envenomation and outcome (analysis of 685 cases). Am J Trop Med Hyg. 2010;83(5):1084-92.

14. Jalali A, Pipelzadeh MH, Sayedian R, Rowan EG. A review of epidemiological, clinical and in vitro physiological studies of envenomation by the scorpion Hemiscorpius lepturus (Hemiscorpiidae) in Iran. Toxicon. 2010;55(2-3):173-9.

15. Sitprija V. Animal toxins and the kidney. Nat Clin Pract Nephrol. 2008;4(11):616-27.

16. Jalali A, Pipelzadeh MH, Seyedian R, Rahmani A H, Omidian N. In vivo pharmacological study on the effectiveness of available polyclonal antivenom against Hemiscorpius lepturus venom. J Venom Anim Toxins incl Trop Dis. 2011;17(2):142-9.

17. Jalali A, Pipelzadeh MH, Seyedian R, Rahmani A H, Omidian N, Mahdavinia M. In vitro pharmacological study upon the effectiveness of available antivenom against Hemiscorpius lepturus venom. Jundishapur J Nat Pharm Products. 2011;6(1):1-8.

18. Ismail $M$. The scorpion envenoming syndrome. Toxicon. 1995;33(7):825-58.

19. Possani LD, Becerril B, Delepierre M, Tytgat J. Scorpion toxins specific for $\mathrm{Na}+$-channels. Eur J Biochem. 1999;264(2):287-300.

20. Borchani L, Sassi A, Shahbazzadeh D, Strub JM, TounsiGuetteti H, Boubaker MS, et al. Heminecrolysin, the first hemolytic dermonecrotic toxin purified from scorpion venom. Toxicon. 2011;58(1):130-9.

21. Jalali A, Pipelzadeh MH, Taraz M, Khodadadi A, Makvandi M, Rowan EG. Serum TNF- $\alpha$ levels reflect the clinical severity of envenomation following a Hemiscorpius lepturus sting. Eur Cytokine Netw. 2011;22(1):5-10.

22. Tambourgi DV, Pedrosa MF, de Andrade RM, Billington SJ, Griffiths M, van der Berg CW. Sphingomyelinases $\mathrm{D}$ induce direct association of $\mathrm{Clq}$ to the erythrocyte membrane causing complement mediated autologous haemolysis. Mol Immunol. 2007;44(4):576-82.

23. Bhakdi S, Tranum-Jensen J. Damage to cell membranes by pore-forming bacterial cytolysins. Prog Allergy. 1988;40:1-43.

24. Sano-Martins IS, Tomy SC, Campolina D, Dias MB, Castro SCB, Sousa-Silva MCC, et al. Coagulopathy following lethal and non-lethal envenoming of humans by the South American rattlesnake (Crotalus durissus) in Brazil. Q J Med. 2001;94(10):551-9. 